Advances in Data Science and Adaptive Analysis

Vol. 8, No. 2 (2016) 1650010 (26 pages)

(C) The Author(s)

DOI: $10.1142 / \mathrm{S} 2424922 \mathrm{X} 16500108$

\title{
On the EMD Sifting Property and Instantaneous Parameters
}

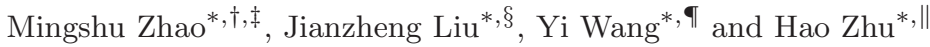 \\ * The Department of Electrical Engineering, Tsinghua University \\ State Key Lab of Control and Simulation of Power Systems and \\ Generation Equipments, Beijing, Haidian District, P. R. China \\ $\dagger$ Sichuan Energy Internet Research Institute, Tsinghua University \\ Beijing, Haidian District, P. R. China \\ $\ddagger$ z.mingshu1008@outlook.com \\ §liujianzheng@263.net \\ 『wangyixiaopang@163.com \\ "|andychuxitu@163.com
}

Received 14 August 2015

Revised 30 June 2016

Accepted 8 July 2016

Published 29 September 2016

\begin{abstract}
Recently proposed Hilbert-Huang transform (HHT), consisting of empirical mode decomposition (EMD) and Hilbert spectrum analysis (HSA), has been proved to be an effective approach in both scientific researches and engineering applications. However, this method is still empirical because of lacking rigorously mathematical foundation. This paper primarily focuses on providing a mathematical contribution on its sifting characteristics and instantaneous features. Firstly, the theory of the original methods as well as their advantages and restrictions are briefly reviewed. Secondly, we conduct indepth investigations of the instantaneous parameters (IPs) and sifting ability. Thirdly, we proposed a new EMD stopping criterion, determined an optimal number of sifting iterations, employed a new masking signal to fix the mode mixing problem and investigated into the sifting property according to the extremum distribution. Finally, comparative studies, simulations and real data analyses depending on the proposed method are presented to demonstrate the validity of the novel research. The simulations illustrate that the typically defined intrinsic mode functions (IMFs) are not perfectly symmetric with zero-mean, there is still no rigorous mathematical standard to determine the "watershed" between mono and multi-component IMFs. The comparative researches indicate that unlike the prism property of the Fourier transform (FT) and the mathematical microscope property of the wavelet transform (WT), the ultimate goal of the HHT is to work as raindrops.
\end{abstract}

Keywords: Empirical mode decomposition (EMD); Hilbert-Huang transform (HHT); instantaneous parameters (IPs); intrinsic mode functions (IMFs); leakage current (LC).

This is an Open Access article published by World Scientific Publishing Company. It is distributed under the terms of the Creative Commons Attribution 4.0 (CC-BY) License. Further distribution of this work is permitted, provided the original work is properly cited. 


\section{Introduction}

The extraction of hidden physical meanings is critical for determining the underlying mechanisms involved in any given signal. Frequency analysis based on the Fourier transform (FT), time-frequency analysis such as the STFT and Cohenclass quadratic distributions, and time-scale analysis based on the wavelet transform (WT) are often applied to investigate the hidden properties of real signals. Unfortunately, the FT can represent the meaningful spectrum property of only linear and stationary processes; the STFT and WT require a predefined basis (such as a mother wavelet); moreover, these integral transform techniques suffer from the Heisenberg uncertainty principal, which limits their ability to accurately measure time and frequency properties simultaneously. A series of relatively ideal TF representations are achieved by the Wigner-Ville distribution (WVD) and other techniques derived from it, which are members of Cohen's class of quadratic distributions. Because of the bi-linearity of the WVD, it suffers from serious cross-terms and auto-terms when applied to multi-frequency signals or mono-frequency signals with a nonlinear time-varying frequency.

The Hilbert-Huang transform (HHT), developed by Huang et al., is the combination of empirical mode decomposition (EMD) and Hilbert spectral analysis (HSA) [Huang et al. $(1998,1999)]$. The key part is EMD, by which any multi-component signal can be decomposed into a set of intrinsic mode functions (IMFs) that admit a well-defined Hilbert transform (HT). Since its introduction, the HHT has been used in a wide range of applications: power quality analysis [Afroni et al. (2013)]; signal filtering [Boudraa and Cexus (2007)]; image processing [Linderhed (2009)]; geophysical studies [Huang and $\mathrm{Wu}(2008)]$ and structure damage detection [Salvino et al. (2005)] et al. Together with numerical engineering applications [Huang and Attoh-Okine (2005)], many simulations have been performed to provide a better understanding of the theoretical framework of EMD and instantaneous parameters (IPs) [Huang (2014)], for example: the filter bank property of EMD [Flandrin et al. (2004); Wu and Huang (2004)], B-spline for convergence analysis [Riemenschneider et al. (2005)], a more robust method named EEMD [Wu and Huang (2009)], CEEMD [Yeh et al. (2010)], multi-dimensional extension of EEMD [Wu et al. (2009)], synchrosqueezed WT inspired by EMD [Daubechies et al. (2009)], a PDE-based sifting process [Deléchelle et al. (2005)], the Hilbert spectral property [Huang et al. (2011)] and a variety of tests on IMF characteristics [Wu and Huang (2005); Sharpley and Vatchev (2006); Qian (2010); Diop et al. (2010)] and IFs [Huang et al. (2009)]. The MATLAB codes are provided in [Auger et al. (1998); Rilling and Goncalves (2008); Buckheit et al. (2005)].

The remainder of this paper is organized as follows. Section 2 presents the HHT algorithm in a nutshell. In Sec. 3, we introduce the main concept of extracting instantaneous features. The performance of the proposed concepts is assessed in Sec. 4. Section 5 gives the experimental investigations and simulations. Section 6 provides the conclusions of this work. 


\section{Hilbert-Huang Transform}

The HHT is a two-step algorithm, consisting of EMD and HSA.

\section{1. $E M D$}

EMD [Huang et al. $(1998,1999)$ ] is a type of self-adapted signal decomposition method. Through the "sifting" process, any multi-component signal of length $N$ can be decomposed into a small number of $\left(M<\log _{2}^{N}\right)$ zero-mean amplitude and frequency modulated (AM-FM) oscillating modes (or, in general, mono-component signals), termed IMFs [Huang et al. (1998)], from which corresponding analytic signals and meaningful IPs can be determined. IMFs are local, unique, complete and nearly orthogonal to each other. An ideal IMF is any function satisfying the following conditions:

(a) Narrow Band Requirement: Within the entire dataset, the number of extrema and the number of zero-crossings must either be equal or differ at most by one.

(b) Local Zero-Mean Condition: At any point, the average (local mean) of the smooth envelopes defined by the local maxima and local minima is zero.

To be a perfect IMF, the absolute value of two envelopes must be the same polynomial function over the entire data range, that is, $\operatorname{env}_{\text {mean }}(t)=\left[\operatorname{env}_{\max }(t)+\right.$ $\left.\operatorname{env}_{\min }(t)\right] / 2=0$. A function that is required to satisfy only condition (a) will be called a weak-IMF [Sharpley and Vatchev (2006)]; this function is generally called an IMF in many studies, satisfying $\operatorname{env}_{\text {mean }}(t) \leq \epsilon$ for some prescribed $\epsilon>0$.

The sifting process (Fig. 1) serves two purposes: to eliminate riding waves and make the wave profiles more symmetrical [Huang et al. (1998); Huang (2014)]. The approach is described by Huang et al. [Huang et al. (1998)]. After the sifting process, any signal can be represented as a combination of IMFs and a residue. Based on the EMD approach, the first IMF component contains the highest oscillation frequencies found in the original signal, typically noise.

$$
x(t)=\sum_{i=1}^{n} \operatorname{imf}_{i}(t)+r_{n}(t)=\sum_{j=1}^{p} \operatorname{imf}_{j}(t)+\sum_{k=p+1}^{q} \operatorname{imf}_{k}(t)+\sum_{l=q+1}^{n} \operatorname{imf}_{l}(t)+r_{n}(t),
$$

where $r_{n}(t)$ is the residual trend, which is typically a monotonic function representing either the mean trend or a constant, and $\operatorname{imf}_{i}(t)$ is the $i$ th IMF, which is the AM-FM function of time, or simply a mono-component signal [Boashash (1992c)].

$$
\operatorname{imf}_{i}(t)=\Re e\left[A_{i}(t) e^{j \int \omega_{i}(t) d t}\right], \quad \omega_{i}(t)>0 .
$$

In contrast to the FT, which separates data into harmonics with a fixed amplitude, frequency and phase, EMD decomposes data into IMFs (including details and local trends), each of which is approximately a zero-mean function, albeit not necessarily with a constant frequency. 


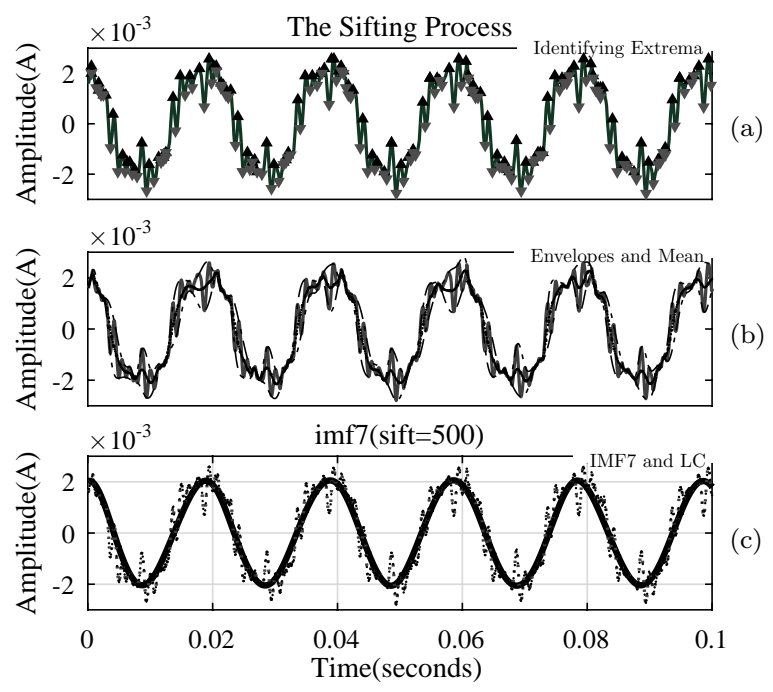

Fig. 1. Illustration of the EMD sifting process: (a) identifying maxima and minima from the analyzed data, (b) constructing the upper and lower envelopes by spline-fitting and calculating the average(local mean) of both envelopes, and (c) comparison of IMF7 and the original signal.

Unlike the conventional TF methods, EMD is intuitive, direct and adaptive, with an entirely data-driven property [Huang and Shen (2005)]. Hence, it is suitable for analyzing signals from nonstationary and nonlinear processes.

\section{2. $H S A$}

Once the EMD process has finished, the second step of the HHT is performed to estimate the IPs of the IMFs. Theoretically, one can easily employ the HT to compute the IF of any given energy-limited real-valued signal $x(t) \in L^{2}$. Through the HT, the complex conjugate of $x(t)$ can be determined as [Hahn (1996)]

$$
y(t)=\operatorname{HT}[x(t)]=\frac{1}{\pi} P \int_{-\infty}^{+\infty} \frac{x(\tau)}{t-\tau} d \tau,
$$

where $P$ is the principal value of the singular integral (Cauchy principal value).

Gabor [1946] defined the instantaneous amplitude and IF through an analytic signal $z(t)=x(t)+j y(t)=a(t) e^{j \varphi(t)}$.

$$
\left\{\begin{array}{l}
a(t)=\sqrt{x^{2}(t)+y^{2}(t)} \\
\varphi(t)=\arctan [y(t) / x(t)]
\end{array}\right.
$$

where $a(t)$ is the instantaneous amplitude and $\varphi(t)$ is the phase function. With these definitions, one can simply obtain the IF by passing the phase function through a differentiation process.

$$
\omega(t)=\frac{d \varphi(t)}{d t}=\frac{x(t) y^{\prime}(t)-y(t) x^{\prime}(t)}{a^{2}(t)}=2 \pi f(t) .
$$


Physically, the HT of signal $x(t)$ is equal to the convolution of $x(t)$ with $1 / \pi t$. Due to the rapid decay of $1 /(t-\tau)$, we can explore the local rather than global parameters, which are defined by Fourier spectral analysis. Unfortunately, the IF $f(t)$ is a single value function of time; that is, at any given time, there exists only one IF value for a given signal. Therefore, it is limited to mono-component signals [Huang et al. (1998)], which is the main reason why Huang et al. proposed the EMD to decompose data into IMFs. For multi-component signals with more than one frequency component at a given time, directly estimating the IF will lead to a meaningless result. For example, the IF of any two-tone-formed signal with the same amplitude is the average of these IFs (due to amplitude modulation) (Fig. 2).

$$
\left\{\begin{array}{l}
x(t)=a(t) \cos \varphi_{n}(t)+a(t) \cos \varphi_{m}(t), \\
z(t)=2 a(t) \cos \left[\frac{\varphi_{m}(t)-\varphi_{n}(t)}{2}\right] e^{\left[j \frac{\varphi_{m}(t)+\varphi_{n}(t)}{2}\right]} .
\end{array}\right.
$$

Employing the HT with all IMFs will yield a time-frequency energy spectrum, called the Hilbert spectrum $\operatorname{HS}(t, \omega)$ [Huang et al. (1998); Huang (2014)]. Whether the residue is excluded or included is determined by its form (a local trend or low-frequency (LF) component):

$$
\operatorname{HS}(t, \omega)=\Re e\left[\sum_{k=1}^{n} a_{k}(t) e^{j \int \omega_{k}(t) d t}\right] .
$$

The IMF represents a generalized Fourier expansion and the mathematical expression of EMD in the time-frequency energy domain appears to be a

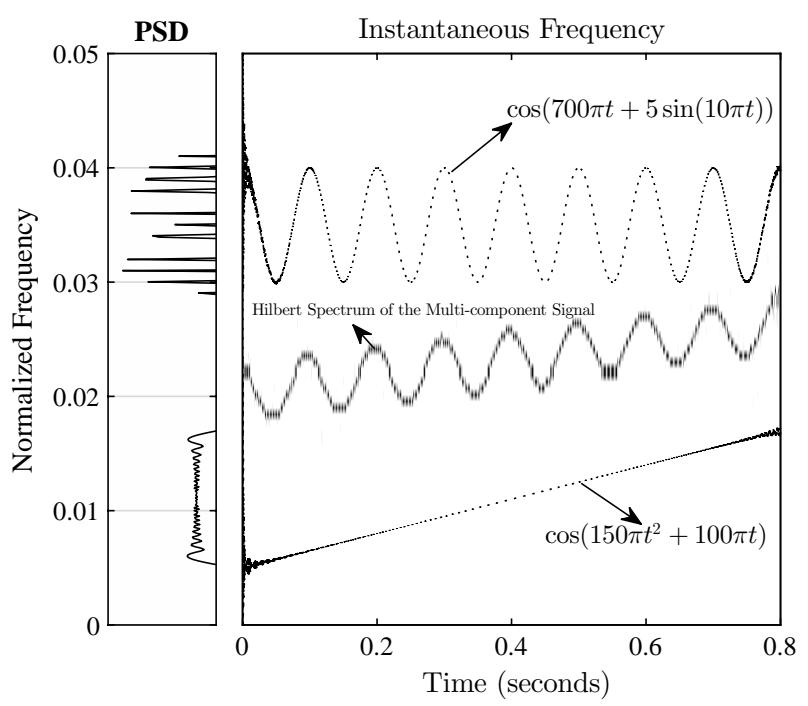

Fig. 2. PSD and IFs of a nonlinear signal with linear-FM and sine-frequency-modulated components; the IF of the signal with the same amplitude is the average of these IFs. 
generalization of the Fourier spectrum [Wu and Huang (2010)], due to the additional dimension $t$.

\section{Instantaneous Feature Extraction}

Historically, the Bedrosian theorem [Bedrosian (1963)] and Nuttall theorem [Nuttall (1966)] have highlighted the deficiencies of the conventional HT. The Bedrosian theorem states that $\operatorname{HT}[a(t) \cos \omega(t)]=a(t) \sin \omega(t)$ only if the spectra of $a(t)$ and $\cos \omega(t)$ disjointed, and the Nuttall theorem demonstrates that $\mathrm{HT}[\cos \omega(t)]$ is not necessarily $\sin \omega(t)$ for any $\omega(t)$. These restrictions have been overcome by the normalized HT (NHT) and direct quadrature (DQ) approaches. Here, we focus on the Hilbert property of nonlinear intra-wave FM signals; see Fig. 3. In contrast to inter-wave frequency modulation, Huang et al. focused on nonlinear dynamics by intra-wave frequency modulation, $x(t)=\cos (\omega t+\epsilon \sin (\omega t))$, which satisfies the following nonlinear differential equation [Huang et al. (1998)].

$$
\frac{d^{2} x}{d t^{2}}+x(t)(\omega+\epsilon \omega \cos \omega t)^{2}=\epsilon \omega^{2} \sin \omega t\left[1-x^{2}(t)\right]^{1 / 2},
$$

where $\omega=2 \pi f$ is a constant carrier frequency and $\epsilon$ is a real parameter termed as the modulation index.

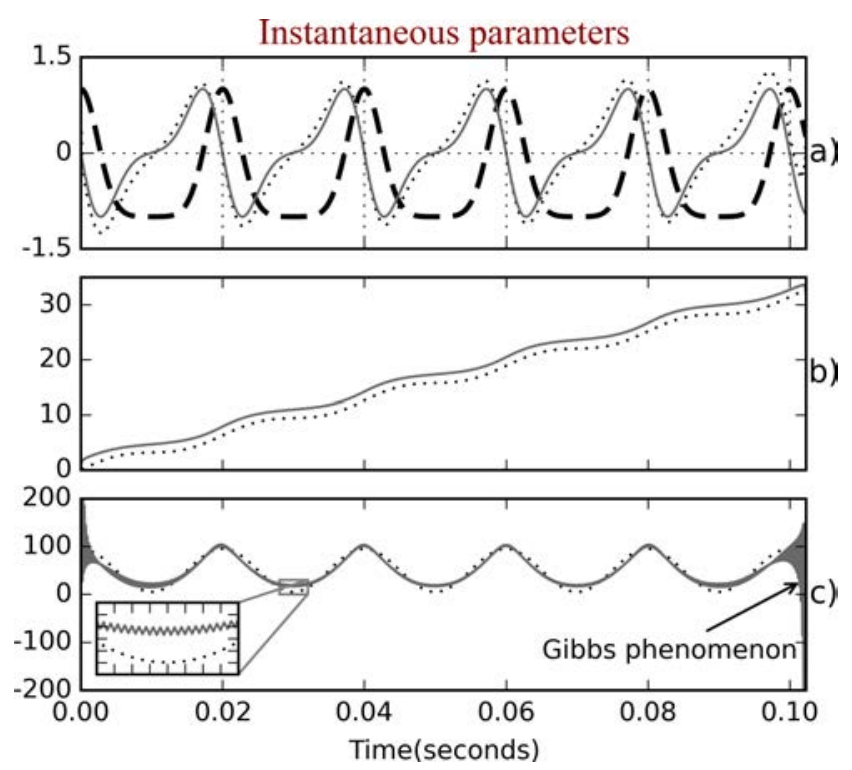

Fig. 3. IPs of the FM signal $\cos (\omega t+\epsilon \sin (\omega t)), \omega=100 \pi, \epsilon=0.8$, (a) real and imaginary parts of the analyzed signal: nonlinear data (dashed line), ideal imaginary part (dotted line) and imaginary part derived from the HT (solid line), (b) phase function: ideal phase (dotted line) and phase derived from the HT (solid line), and (c) IF: ideal IF (dotted line) and estimated IF (solid line). The example shows that the HT has other mathematical problems in addition to the boundary effects: for a certain nonlinear circumstance, the HT method is still insufficient to present ideal IPs even for the most reasonable definitions of IMFs. 
The HT has an end effect (or Gibbs phenomenon) because its computation derives from the FFT, due to an incomplete data periodicity at the boundaries [Feld$\operatorname{man}(2011)]$.

In addition, when working with nonlinear processes, such as harmonic distortion (more precisely, intra-wave modulation), the HT has other mathematical issues. Because of serious nonlinearity, constructing the imaginary part of this ideal monocomponent signal via HT operation may not yield a perfect quadrature from the data because the HT is equivalent to eliminating negative frequencies of the spectrum [Boashash (1992a,b)], and the error between the ideal IF and estimated IF becomes large with increases in the index $\epsilon$.

Another function called the Teager energy operator (TEO) has been applied to calculate IPs that are entirely dependent on differentiations. The details of this function are presented in [Huang et al. (2009)] and [Kvedalen (2003)]. One can easily obtain both the amplitude and frequency through the TEO:

$$
\left\{\begin{array}{l}
a=\Psi[x(t)] / \sqrt{\Psi[\dot{x}(t)]}, \\
\omega=\sqrt{\Psi[\dot{x}(t)] / \Psi[x(t)]},
\end{array}\right.
$$

where $\Psi[x(t)]=\dot{x}^{2}(t)-x(t) \ddot{x}(t)$ is the energy operator.

By using an iterative normalization scheme, any AM-FM signal $x(t)=$ $a(t) \cos (\omega t+\epsilon \sin \omega t)$ can be separated into envelope $a(t)$ (AM) and carrier $\cos (\omega t+\epsilon \sin \omega t)(\mathrm{FM})$ parts. Hence, a simpler frequency function can be determined as

$$
\omega=\sqrt{\Psi[x(t)]}
$$

Thus, one can obtain the energy operator from the FM part:

$$
\Psi[x(t)]=(\omega+\epsilon \omega \cos \omega t)^{2}-\epsilon \omega^{2} \sin \omega t \sin (\omega t+\epsilon \sin \omega t) \cos (\omega t+\epsilon \sin \omega t) .
$$

The equation shows that the IF of an intra-wave modulated signal through the TEO is an oscillating function and that the IF is only meaningful when $\epsilon=0$. Although the normalized TEO is a simple and direct method to compute the IF, it still has some disadvantages: first, it is only suitable for mono-component signals; second, it is highly sensitive to the signal boundaries; and third, it is problematic to analyze nonlinear signals.

The DQ technique can provide the correct phase function as long as the FM part of the analyzed data is in the form of $\cos [\omega(t)]$. The ideal IF can be obtained by using the arctangent (arccos) and unwrapping methods unless the imperfect normalization. However, there may exist some periodic vibrations in the IF in certain cases.

An alternate method is based on quadratic time-frequency distributions, and as the basic joint, WVD provides a high signal energy concentration in the time and frequency domains. The first moment of the WVD with respect to frequency yields the weighted mean local frequency.

The bi-linearity introduces cross-terms and auto-terms when dealing with multi-frequency signals or mono-frequency signals with a nonlinear time-varying 
frequency. One can progressively alleviate the unnecessary terms by introducing appropriate kernels (such as smoothed pseudo-WVD (SPWVD)).

\section{Improvements to EMD}

Although the HHT is a relatively efficient approach to analyze nonstationary and nonlinear signals, it still has some deficiencies for real data analysis.

The first limitation of this method is its rather LF resolution [Wu and Huang (2004); Flandrin et al. (2004)]; conventional EMD is unable to distinguish two tones with a frequency ratio higher than 0.67 [Rilling and Flandrin (2008)] or, more simply, within one octave [Deering and Kaiser (2005)]. Another limitation of the method is that its direct application in the intermittency of real-world data may lead to mode mixing [Huang and Shen (2005)]; because an IMF is a function with time-varying amplitude and frequency, the mode mixing problem is inevitable. In addition, the boundary conditions also have an effect on the IMFs. If left unattended, the boundary conditions will produce large swings on the ends, which will progressively corrupt the LF IMFs. Huang et al. adopted a method of adding characteristic waves at the end [Huang et al. (1998)], whereas Rilling proposed a method of mirroring the extrema at the boundaries [Rilling et al. (2003)]. Such methods are empirical but critical to reduce the errors at the boundaries because they aim to increase artificial extrema on the boundaries to avoid the divergence of the spline functions (through extrema interpolation schemes). Among the variety of techniques, Rilling's method is adaptive, robust and simple to apply.

\subsection{A new stopping criterion for EMD}

For the majority of signals generated from a power system, the most important part is typically the LF component. Leakage current (LC), often presenting as a dominant frequency of approximately $50 \mathrm{~Hz}$ (or $60 \mathrm{~Hz}$ ), called the fundamental frequency, is distorted by higher harmonics with relatively lower amplitude. The frequencies of these components often lie within one octave.

The sifting process starts by extracting the highest frequency. Therefore, by employing EMD on the LC, the last IMF should be the fundamental component; therefore, we proposed a new stopping criterion based on the energy of the fundamental component. By computing the energy once an IMF is extracted, the sifting is stopped when the energy ratio between the IMF and the original signal is sufficiently large. The new stopping criterion is suitable for relatively stationary LCs and it will lose power when many intermittencies occur. Accordingly, this paper set the maximum number of IMFs to 9 based on prior knowledge of LC.

\subsection{Optimal number of sifting iterations}

Over-sifting will reduce the IMF to a constant-amplitude FM function [Huang et al. (2003)]. Under-sifting would not produce satisfactory IMFs. The number of sifting 
steps is related to the sifting stopping criterion. There are three widely used mathematical stopping criteria: the Cauchy-type convergence criterion [Huang et al. (1998)], the S-number-based criterion [Huang et al. (1999)] and the mean value criterion [Rilling et al. (2003)].

The major flaw of the Cauchy-type convergence criterion is that it is unrelated to the definition of the IMF. The deficiency of the S-number-based criterion is that the S-number is a difficult parameter to determine a priori for an unknown dataset. The mean value criterion is meaningful because it guarantees globally small oscillations in the mean while also considering locally large excursions; however, different parameters will lead to different results. Furthermore, neither of these stopping criteria can lead us to obtain a meaningful result when the frequencies of different components lie within one octave.

When the frequencies of two tones are far apart, a small number of sifting steps will separate them effectively, but when they lie within one octave, the synthetic signal is more likely to behave as an AM single tone. This behavior can be explained as follows: any two harmonic signals and their equivalent form are

$$
\cos \omega_{1} t+\cos \omega_{2} t=2 \cos \left[\frac{\omega_{1}-\omega_{2}}{2} t\right] \cos \left[\frac{\omega_{1}+\omega_{2}}{2} t\right] .
$$

This function demonstrates that the interpretation of this signal is not unique: two co-existing tones with the same amplitude could be considered as an AM single component when their frequencies are sufficiently close (often within one octave); hence, one may observe the prominent "beat effect" [Majumdar (2007)], which shows that the sum of two IMFs can yield a third IMF.

It has been shown that when the sifting number is small, EMD will behave as a dyadic filter bank [Flandrin et al. (2004); Wu and Huang (2004)]. The synthetic signal is considered as an AM wave with a frequency that is the mean frequency of the prescribed sinusoidal waves.

As the sifting number increases, the filter bank will have narrower bands, and thus, the ability to separate components increases as well [Wu and Huang (2010)]. As noted by Wu and Wang [Wu and Huang (2010); Wang et al. (2010)], the EMD process can act as a dyadic filter when the iterations of the sifting process are relatively small and as a nondyadic filter if the iteration number is high; an infinite number of iterations in sifting results in a constant amplitude of the envelope [Wang et al. (2010)]. Eventually, the amplitude modulation is smoothed and the amplitudes would become uniform with only the frequency retaining some modulation [Wu and Huang (2010)], thus approaching the result of Fourier decomposition. Therefore, theoretically, EMD can achieve an arbitrarily high frequency resolution based on a sufficient number of iterations, explained by the fact that any signal cannot have both maxima and minima at a single point; however, this fine resolution is achieved at the cost of information leakage. Twenty thousand sifting iterations can separate this synthetic signal but at the cost of time consuming and information losing (Fig. 4). Moreover, because EMD is an iterative method, the error caused by the 

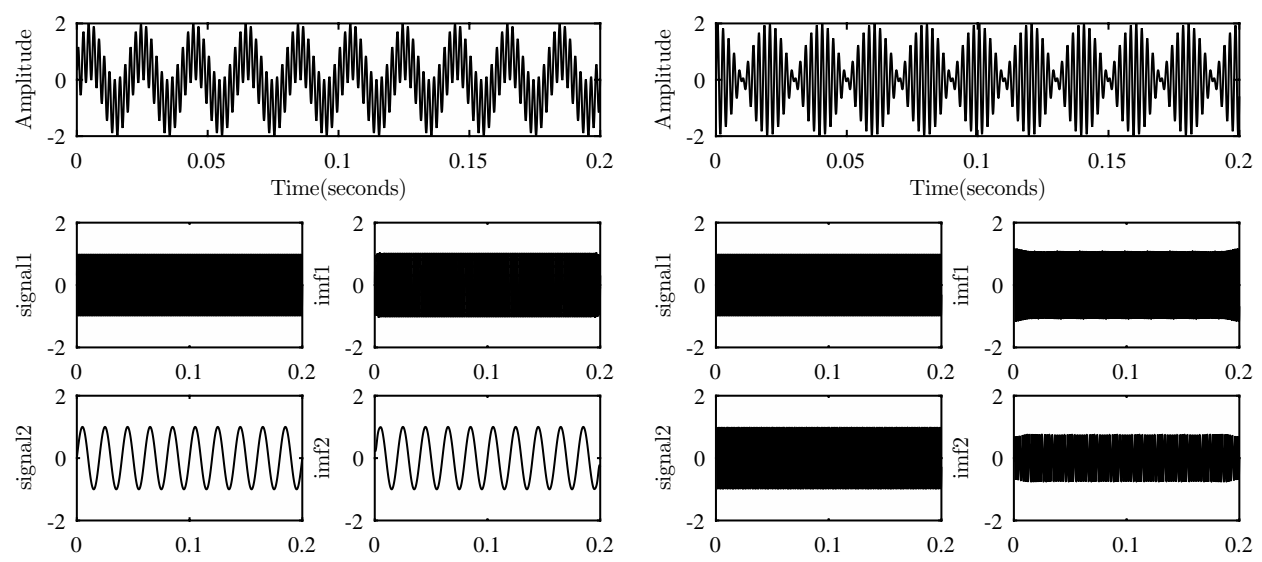

(a)

(b)

Fig. 4. EMD of two tones: $\omega=1000 \pi$, the signal of (b) is already a weak-IMF, so EMD with a small number of sifting iterations incorrectly identifies the multi-component signal as a single tone, whereas large amount of iterations can lead to correct separation at the cost of time consumption and information leakage. (a) $f=0.1$, sifting 10 and (b) $f=0.9$, sifting 20000 .

end effect will accumulate with each sifting iteration; this accumulation leads to corruption in the IMF components; thus, the number of iterations should be kept low but not so low that components whose frequencies lie within one octave cannot be separated. In other words, there is a trade-off between frequency resolution and information leakage; thus, an optimal sifting iteration is needed to obtain the best IMFs.

Our main goal is to choose the optimal number of sifting iterations at which EMD can efficiently separate the given signal that has frequencies lying within one octave and can obtain the best IMFs with minimal leakage at the same time.

Consider the two-tone signal illustrated by Rilling [Rilling and Flandrin (2008)]

$$
x(t)=a_{1} \cos \left(2 \pi f_{1} t+\varphi_{1}\right)+a_{2} \cos \left(2 \pi f_{2} t+\varphi_{2}\right) .
$$

with $f_{1} \ll f_{s}$ and $f_{2} \ll f_{s}$, where $f_{s}$ is the sampling frequency. Let the relative parameters $a=a_{2} / a_{1}, f=f_{2} / f_{1}$ and $\varphi=\varphi_{2}-\varphi_{1}$; then, we can obtain the simple model:

$$
x(t ; a, f)=\cos (2 \pi t)+a \cos (2 \pi f t+\varphi) .
$$

Moreover, if $f \in(0,1)$, the $\cos (2 \pi t)$ component will be regarded as the higherfrequency (HF) component, and the $a \cos (2 \pi f t+\varphi)$ component will be the lowerfrequency (LF) component. To demonstrate the sifting ability, we do not consider the relative phase $\varphi$; indeed, if considering the relative phase, the situations will become rather complex. Consider the resolution ratio of the two tones:

$$
r(a, f) \triangleq \frac{\left|\operatorname{imf}_{1}(t)-\cos (2 \pi t)\right|^{2}}{|a \cos (2 \pi f t)|^{2}} .
$$




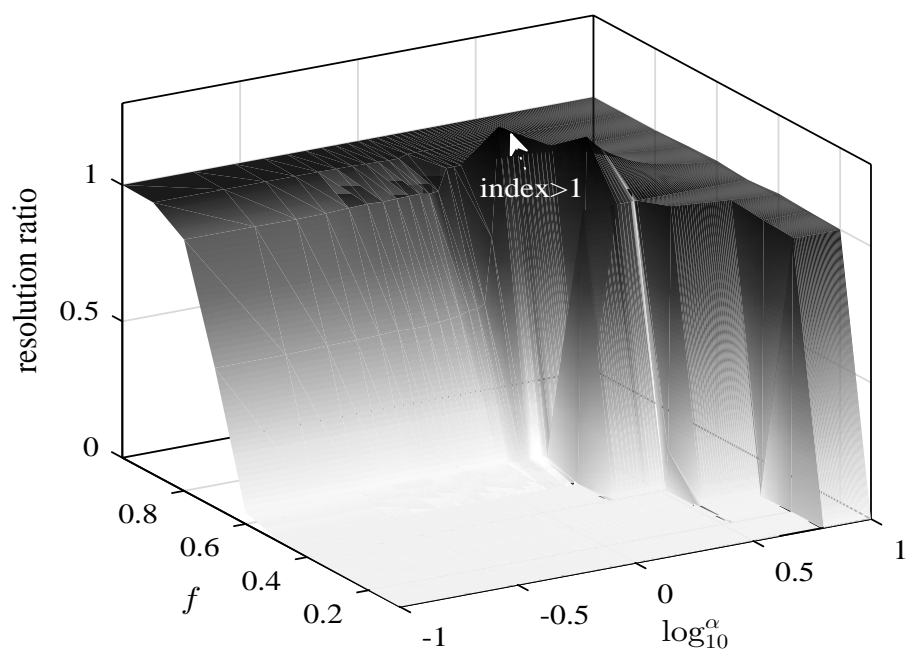

Fig. 5. Reproduction of Rilling's research: performance measure of separation for two-tone signals, sifting 10. When the LF component has a larger amplitude - that is $a>1$ - and the two-harmonic signal has close frequencies $f>0.5$, regardless of the sifting iterations, one cannot obtain the correct result, which results in a resolution ratio larger than 1 . This phenomenon is related to the extremum distribution, mainly because some extrema of the HF component are covered by the LF component.

A zero value for (18) indicates perfect separation of the two components, whereas a value near 1 occurs when the two harmonics are poorly separated (see Fig. 5) [Rilling and Flandrin (2008)].

The sifting ability behaves similarly when $a \leq 1$ (Fig. 5). Hence, when considering the resolution ratio influenced by sifting iterations, the amplitude ratio $a$ should be maintained at 1 to avoid the influence caused by different amplitude ratios; we can then obtain the relationship between resolution ratios and sifting iterations.

For every specific frequency ratio, the sifting iterations and resolution ratios follow an exponential distribution (see Fig. 6(a)):

$$
r(s)=e^{-\tau s},
$$

where $r(s)$ represents the approximating curve and $s$ is the number of sifting iterations. Here, we can obtain the coefficient $\tau$ from any known sifting iteration

$$
\tau=\frac{-\ln r(s)}{s} .
$$

Finally, we can obtain the frequency ratio of all specific iterations. For a frequency ratio less than $0.7,100$ times of sifting is sufficient, but a HF ratio necessitates more sifting iterations to obtain the approximating curve (see Fig. (6b)):

$$
r(s)=e^{-\tau s}, \quad f<0.7, s \in[1,100] .
$$

Through this function, we can select an optimal sifting number. Indeed, 100 times of sifting can easily separate two tones whose frequency ratio lies under 0.7 , 


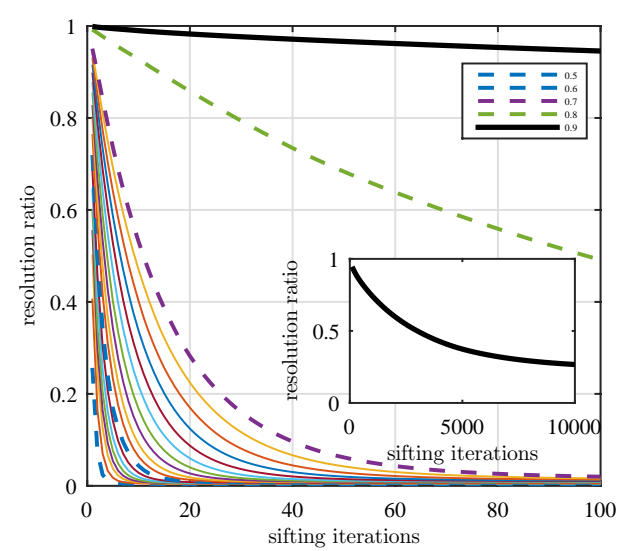

(a)

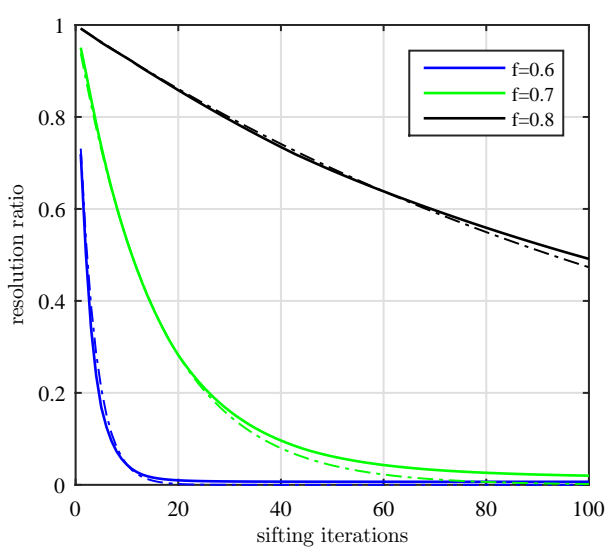

(b)

Fig. 6. EMD of two tones with different sifting iterations, the sifting iterations and resolution ratios follow an exponential distribution. (a) Relationship between sifting iterations and resolution ratios and (b) Experimental results (solid line curve) and theoretical predictions.

whereas the frequency resolution of EMD decreases at higher IF values. For real data, such as LC, the HF harmonics often have a frequency ratio over 0.8 . Therefore, here, we set the sifting iterations to 500 to separate the HF harmonics.

\subsection{Multi-component or mono-component: About extrema}

All IMFs are closely correlated to the interpolation function, which defines the upper and lower envelopes.

$$
\begin{aligned}
\operatorname{imf}_{1}(t) & =x(t)-\left[m_{1 k}(t)+\cdots+m_{11}(t)+m_{1}(t)\right] \\
r_{1}(t) & =x(t)-\operatorname{imf}_{1}(t)=m_{1 k}(t)+\cdots+m_{11}(t)+m_{1}(t) .
\end{aligned}
$$

Consequently, other residues are entirely determined by spline functions [Huang and Shen (2005); Wang et al. (2010)]. Because the spline function is closely connected with the extrema, the ability to find extrema related to the components is critical.

Rilling [Rilling and Flandrin (2008)] investigated the extremum distribution of a two-tone signal and reached the conclusion that EMD can extract a component only if it "sees" extrema that are related to it. In other words, EMD cannot distinguish a specific component if no extrema are related to that component. More specifically, if $a f<1$, the extremum rate is identical to the HF component, and this area is "normal" because EMD extracts the highest-frequency component into the first IMF. However, recovering the HF component in the $a f^{2}>1$ area is unlikely because the extremum of LF is the dominant one; thus, this area can be viewed as "abnormal". 


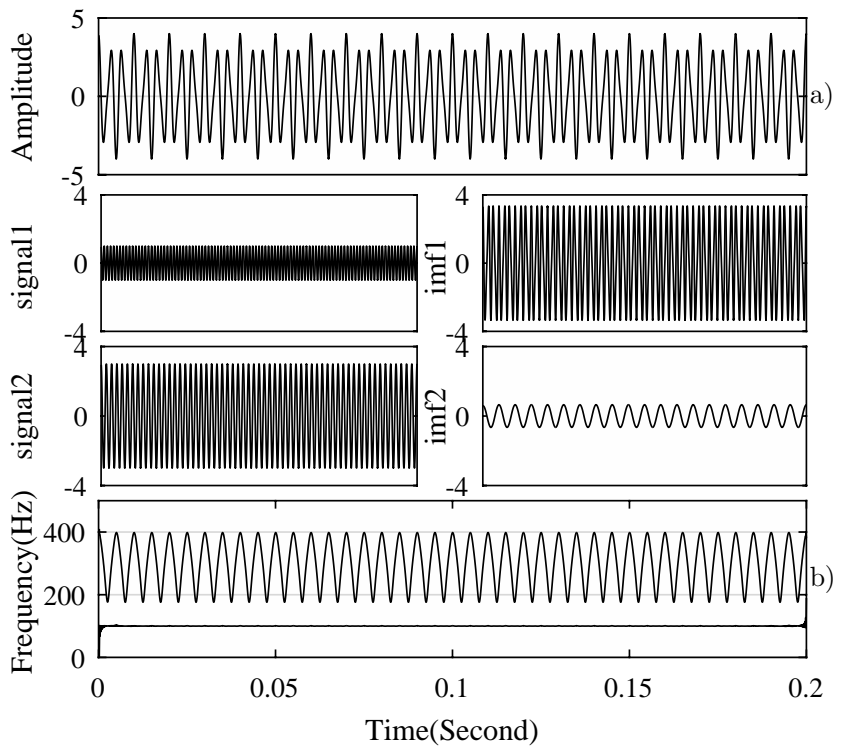

Fig. 7. Two tones act as a harmonic and an FM signal: $\omega=1,000 \pi, a=3, f=0.6$, sifting 100, (a) original signal and (b) instantaneous frequencies of IMFs through the NHT.

\subsubsection{Extrema related to the HF component}

In this case, af $<1$, EMD can seek out all extrema related to the HF component; therefore, theoretically, EMD can separate two components with specific sifting iterations.

\subsubsection{Some extrema related to the HF component covered by LF component}

In certain instances when af $>1$, the EMD of a two-tone signal with different amplitudes will behave as a combination of an FM signal and a pure tone (see Fig. 7) [Laila et al. (2009)]. Because EMD more likely to extract the FM signal as the first IMF due to the extremum distribution, this will lead to a resolution ratio larger than 1 (Fig. 5).

$$
\begin{aligned}
x(t) & =a \cos \left(\omega_{1} t\right)+\cos \left(\omega_{2} t\right), \quad 0.5 \omega_{2}<\omega_{1}<\omega_{2}, a>1 \\
& \cong a \cos \left[\omega_{1} t+\frac{2}{a} \sin \left(\omega_{2}-\omega_{1}\right) t\right]+\cos \left(2 \omega_{1}-\omega_{2}\right) t .
\end{aligned}
$$

\section{Proof.}

$$
\begin{aligned}
a \cos \left[\omega_{1} t+\epsilon \sin \left(\omega_{2}-\omega_{1}\right) t\right] & \cong a \cos \omega_{1} t-a \epsilon \sin \omega_{1} t \sin \left(\omega_{2}-\omega_{1}\right) t \\
& \cong a \cos \omega_{1} t-\frac{a \epsilon}{2}\left[\cos \left(2 \omega_{1}-\omega_{2}\right) t-\cos \omega_{2} t\right] .
\end{aligned}
$$

If $\epsilon=2 / a$, the equation becomes $a \cos \omega_{1} t+\cos \omega_{2} t-\cos \left(2 \omega_{1}-\omega_{2}\right) t$. 


\section{Zhao et al.}

This problem can be fixed by the frequency heterodyne technique or single-phase DQ transform. Unfortunately, the frequency heterodyne method is nonadaptive, and one of its significant drawbacks is the difficulty in selecting an appropriate heterodyne frequency [Senroy et al. (2007)]. Another deficiency is that this approach is only suitable for stationary signals. Moreover, the single-phase DQ transform is nonadaptive and also works only for stationary situations.

\subsubsection{Extrema related to the LF component}

In certain cases, when the extrema are all related to the LF component, $a f^{2} \geq 1$, the EMD decomposition will never distinguish all components. For convenience, consider the frequency ratio of $1 / 2^{n}$ such that:

$$
x(t)=\cos (\omega t)+a \cos \left(\frac{1}{2^{n}} \omega t\right), \quad n \in N .
$$

Let us assume that $x(t)$ admits an extremum at $t=t_{0}$; compute the first derivative of the signal

$$
\begin{aligned}
& \frac{d x\left(t_{0}\right)}{d t}=-\omega \sin \left(\omega t_{0}\right)-\frac{\omega}{2^{n}} a \sin \left(\frac{1}{2^{n}} \omega t_{0}\right)=0, \\
& 2^{n} \sin \left(\omega t_{0}\right)+a \sin \left(\frac{1}{2^{n}} \omega t_{0}\right) \\
& =\sin \left(\frac{1}{2^{n}} \omega t_{0}\right)\left[2^{2 n} \prod_{i=1}^{n} \cos \left(\frac{1}{2^{i}} \omega t_{0}\right)+a\right]=0 .
\end{aligned}
$$

(a) When $|a|>2^{2 n}\left(|a|=2^{2 n}\right.$ has the same extrema)

$$
2^{2 n} \prod_{i=1}^{n} \cos \left(\frac{1}{2^{i}} \omega t_{0}\right)+a>0 .
$$

The extrema are related only to the LF component.

$$
t_{0}=\frac{2^{n} k \pi}{\omega}, \quad k \in N .
$$

Here, we can obtain the upper envelope $e_{\max }(t)$ and lower envelope $e_{\min }(t)$

$$
\begin{gathered}
\left\{\begin{array}{c}
e_{\max }(t)=1+a, \quad k=0,2,4, \ldots, 2 n, \\
e_{\min }(t)=1-a, \quad k=1,3,5, \ldots, 2 n-1,
\end{array}\right. \\
h(t)=x(t)-\frac{1}{2}\left[e_{\max }(t)+e_{\min }(t)\right] \\
=\cos (\omega t)+a \cos \left(\frac{1}{2^{n}} \omega t\right)-1
\end{gathered}
$$

$h(t)$ is already an ideal IMF, so EMD will never distinguish those two tones correctly (Fig. 8(a)). 

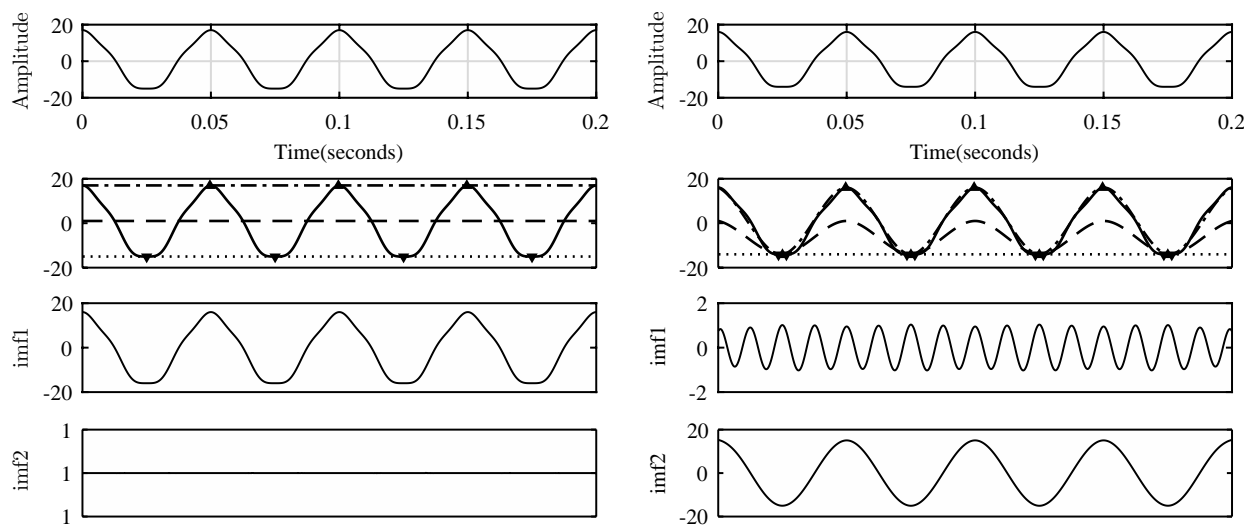

(a)

(b)

Fig. 8. EMD separability measure of a two-tone model, $\omega=160 \pi, n=2$. Whether the LF component can be extracted is entirely dependent on the amplitude ratio $a$. (a) $a=16 \geq 2^{2 n}$ and (b) $a=15<2^{2 n}$.

(b) When $|a|<2^{2 n}$, EMD can extract the HF component because some of the extrema are related to it (Fig. 8(b)).

\subsubsection{Mono-component with different types of extrema}

For a mono-component signal, if EMD can detect different types of extrema, it can decompose the signal into "stronger" mono-components, even perfect IMFs, which are constant-amplitude, FM components. For example, EMD can decompose a single, nonlinear signal into a set of IMFs. Consider the simple example used by Huang et al. [Huang et al. (1998)].

$$
x(t)=\cos (\omega t+\epsilon \sin \alpha \omega t) .
$$

This is an intra-wave modulated signal. Let us assume that $x(t)$ admits an extremum at $t=t_{0}$; compute the first derivative of the signal:

$$
\begin{aligned}
\frac{d x\left(t_{0}\right)}{d t} & =-\left(\omega+\epsilon \alpha \omega \cos \omega t_{0}\right) \sin \left(\omega t_{0}+\epsilon \sin \alpha \omega t_{0}\right)=0 \\
& =\left(1+\epsilon \alpha \cos \omega t_{0}\right) \sin \left(\omega t_{0}+\epsilon \sin \alpha \omega t_{0}\right)=0 .
\end{aligned}
$$

(a) If $|\epsilon| \leq 1 / \alpha$, all extrema are related only to $\sin \left(\omega t_{0}+\epsilon \sin \alpha \omega t_{0}\right)$, hence, $x\left(t_{0}\right)$ is already a rigorous IMF (see Fig. 9(a)). The IF of this mode is

$$
f\left(t_{0}\right)=\frac{\omega}{2 \pi}\left(1+\epsilon \alpha \cos \omega t_{0}\right)
$$

(b) If $|\epsilon|>1 / \alpha$, some extrema are related to $1+\epsilon \alpha \cos \omega t_{0}$; therefore, the EMD algorithm will continue detecting IMFs until all IMFs are stronger or even perfect mono-components (see Fig. 9(b)). Indeed, this type of waveform can be 


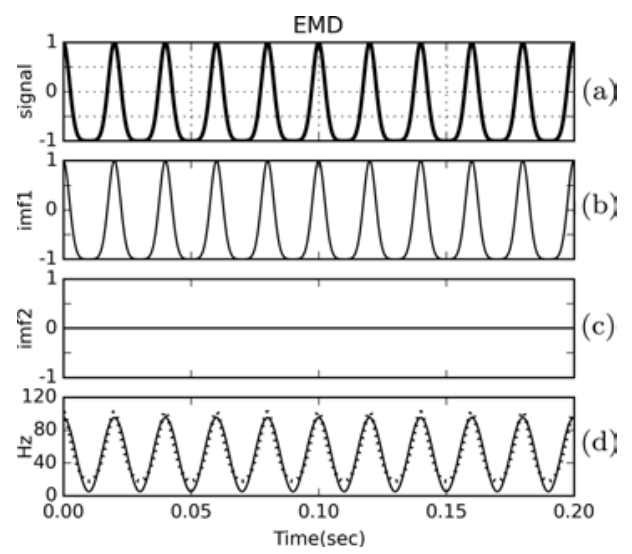

(a)

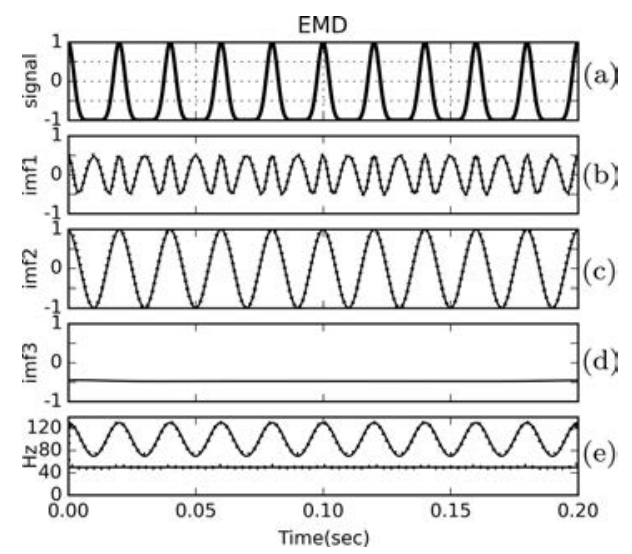

(b)

Fig. 9. EMD of a mono-component signal $\cos (\omega t+\epsilon \sin \alpha \omega t), \omega=100 \pi, \alpha=1$. Original signal (a), elementary components (left: (b) and (c) and right: (b)-(d)) and instantaneous frequencies (left: (d) and right: (e)) of corresponding IMFs with the NHT (dotted line) and normalized DQ (solid line). (b) and (c) (right) represent the decomposed IMFs (solid line) and theoretical functions (dotted line). (a) $\epsilon=0.9, \alpha \epsilon \leq 1$ and (b) $\epsilon=1.1, \alpha \epsilon>1$.

expanded as

$$
\begin{aligned}
x(t) & =\cos \omega t \cos (\epsilon \sin \omega t)-\sin \omega t \sin (\epsilon \sin \omega t) \\
& =\cos \omega t-\epsilon \sin ^{2} \omega t-\frac{\epsilon^{2}}{2} \sin ^{2} \omega t \cos \omega t+R(\omega t) \\
& =\left[-\frac{\epsilon}{2}+R(\epsilon)\right]+\left[1-\frac{\epsilon^{2}}{8}+R(\epsilon)\right] \cos \omega t+\left[\frac{\epsilon}{2}+R(\epsilon)\right] \cos 2 \omega t+R(\omega t),
\end{aligned}
$$

where $R$ is the residue.

In Fig. 9(b), the highest-frequency IMF is an intra-wave modulated component because the higher components mix together and constitute the intra-wave modulated signal. More precisely

$$
x(t) \cong-\frac{1}{2}+\cos \omega t+\frac{1}{2} \cos \left[2 \omega t+\left(\epsilon-\frac{1}{2}\right) \sin \omega t\right], \quad 1<\epsilon \leq 1.5 .
$$

The indexes of DC, harmonic and FM components will change slightly, and EMD will generate other HF modulated IMFs related to $\epsilon$ if $\epsilon>1.5$.

The Fourier series of this FM signal can be defined as

$$
x(t)=\sum_{n} J_{n}(\epsilon) \cos [(n+1) \omega t],
$$

where $J_{n}($.$) is the first type Bessel function of order n$.

When $\epsilon=1$.1, an approximate Fourier series for this signal is

$$
x(t) \cong-0.471+0.720 \cos \omega t+0.471 \cos 2 \omega t+0.137 \cos 3 \omega t,
$$


whereas an approximate EMD expansion for this signal is

$$
x(t) \cong-0.5+\cos \omega t+0.5 \cos (2 \omega t+0.6 \sin \omega t) .
$$

According to FT, the nonlinear phase function of an FM signal leads to a sum of uniform harmonic vibrations with fixed amplitudes, phases and frequencies, whereas EMD is considerably more adaptive (and hence complex) to behave as a linear or nonlinear process due to the adaptive extremum distribution of the target signal.

A mono-component signal is not necessarily a perfect IMF, but a perfect IMF is more likely to be a mono-component signal. However, there is still no rigorously mathematical standard to determine whether a typically defined IMF is a mono-component or multi-component signal or the "watershed" between mono-component and multi-component IMFs, similar to the circumstance of conductors and insulators. Moreover, weak-IMFs can be viewed as the "semi-monocomponents". This conflict between ideal IMFs and estimated IMFs is one of the main reasons why EMD is empirical and ad hoc.

\subsection{Using the new masking signal to alleviate mode mixing}

The intermittency of real-world data is a main cause of mode mixing in EMD, which refers to one IMF containing different scales [Mandic et al. (2013)]. Mode mixing is the major reason that renders the EMD algorithm unstable: any small perturbation may result in a new set of IMFs. The intermittence prevents EMD from extracting any signal with similar scales [Wu et al. (2009)]. The mode mixing problem is also related to the extrema. The mixed IMF contains two or more types of extrema related to different signal components. This problem is inevitable because the EMD process is entirely adaptive and the IMF is a function with time-varying amplitude and frequency. The newest EEMD [Wu et al. (2009)] and CEEMD [Yeh et al. (2010)] largely overcome the false mode mixing problem and provide physically unique decompositions. However, these methods may introduce many meaningless non-IMF components and the sum of IMFs does not necessarily reconstruct the original signal [Tary et al. (2014)], besides, they are extremely time-consuming.

The use of a masking signal is helpful for alleviating the mode mixing problem [Deering and Kaiser (2005)], and the development of this approach is proposed in [Senroy et al. (2007)]; however, the algorithm contains some limitations because it depends on Fourier analysis. This paper proposed a new masking signal to fix this problem by adding large amount of symmetric extrema to the original signal to perform "mode mixing" with the intermittencies. An example used by Huang et al. [Huang et al. (1999)] is presented here (see Fig. 10(a)); the data have a stationary LF part and some HF intermittent oscillations; the original EMD process will lead to mode mixing because the envelope is determined by the LF component and HF intermittent component. Hence, the envelope mean is severely distorted, so the first IMF is the mixture of both the LF fundamental component and the $\mathrm{HF}$ intermittent waves. By using the new masking signal, the frequency is as high 

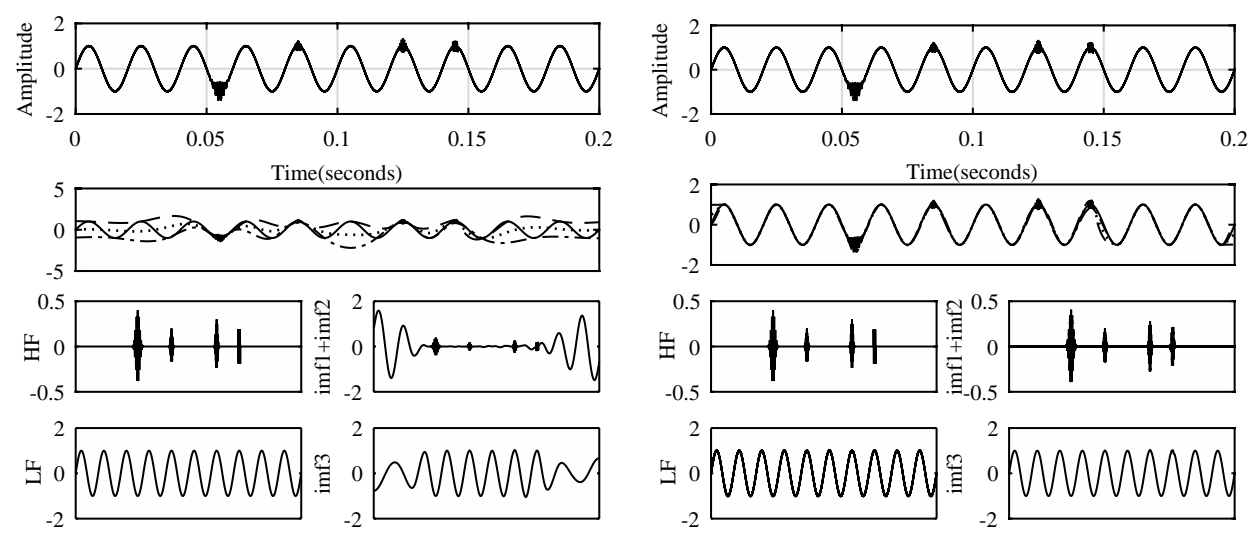

(a)

(b)

Fig. 10. Using the new masking signal technique to eliminate mode mixing phenomenon: masking signal $x(t)=0.01 \cos (8000 \pi t)$. The distorted signal $(-)$, mean $(\cdot)$, lower $(-\cdot)$ and upper (- -) envelopes. (a) EMD with mode mixing and (b) EMD without mode mixing.

as possible to perform "mode mixing" with the HF intermittent waves, but it cannot exceed the Nyquist frequency. Meanwhile, the amplitude need to be sufficiently small because a smaller amplitude results in a smaller error in the IMFs. However, it cannot be arbitrarily small because the artificial signal must be separated from the LF component. As a matter of fact,

$$
x(t)=a \cos \left(2^{n} \omega t\right)+\cos (\omega t), \quad n \in N .
$$

Only when $|a|>1 / 2^{2 n}$ can EMD extract the HF component, so we can determine the frequency and minimum amplitude of the artificial signal. Figure 10(b) demonstrates the validity of this method. This technique is based on the sifting function; in other words, we introduce the HF artificial harmonic to perform "mode mixing" with the intermittent signals. Therefore, if the intermittent signals have wide-spread frequencies, one may need other masking signals with frequencies lying outside one octave.

\section{Simulations and Real Data Analysis}

Among the various TF methods, SPWVD and reassigned spectrogram [Flandrin et al. (2002)] are able to represent the inter and intra-wave properties with a relatively high frequency resolution. The EMD-based SPWVD yields precise frequency resolution as well as high temporal resolution in identifying the intra-wave FM signal with few auto-terms.

LCs measured through a special data acquisition system are employed to further illustrate the characteristics of the novel approach.

There are many well-established techniques for LC analysis, including the FT [Suda (2005)] — Suda investigated the frequency characteristics of LC waveforms through FFT and classified them into six stages to predict flashover; 


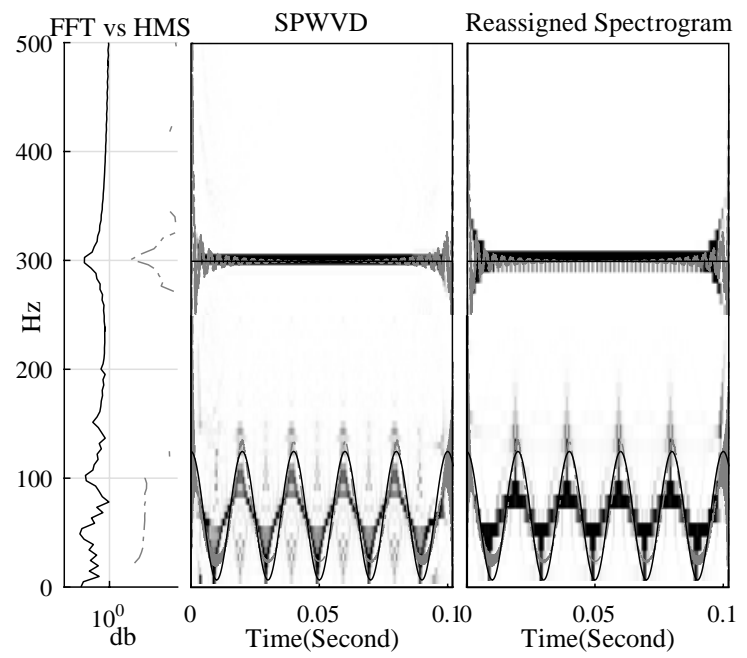

Fig. 11. Nonlinear multi-component signal analysis, $x(t)=0.5 \cos (6 \omega t)+\cos [\omega t+\epsilon \sin (\omega t)], \omega=$ $100 \pi, \epsilon=0.9$, left: FFT spectrum and normalized Hilbert marginal spectrum estimate of the signal (black solid line: FFT; gray dashed line: HMS), middle: SPWVD of two IMFs (black solid line: ideal IF; gray dashed line: IF from NHT), right: reassigned spectrogram of two IMFs (black solid line: ideal IF; gray dashed line: IF from NHT).

STFT [Gao et al. (2006)] and spectrogram [Norddin et al. (2013)] — Norddin et al. discussed the S-transform and spectrogram, and concluded that higher contents of harmonics will indicate the severity of the LC waveform distortion; and the WT [Moula et al. (2013)] — Moula adopted the DWT for LC decomposition and demonstrated that a simultaneous increase in the standard deviation of the specific components indicates the presence of partial arcs. Unfortunately, each of these techniques has its own limitations when working with these nonstationary and nonlinear signals, which are influenced by several factors, including climate, voltage and material factors. Referring to [Suda (2005)], we classified the LC waveforms into seven stages, including flashover (Fig. 12). Due to the limitations, when directly employing the FFT, the frequency characteristics of LCs are misleading when the signals are sharply distorted.

As Fig. 13, the STFT has a fixed resolution, and the width of the windowing function determines whether there is good frequency or time resolution. In contrast, the WT is nonadaptive; that is, the effectiveness of employing WT relies on the choice of the mother wavelet and once it is chosen, one will have to use it to analyze all data. In addition, it suffers from serious spectral leakage [Huang et al. (1998)].

We studied each stage of LC via the original EMD and improved EMD, and EMD-based SPWVD is employed to investigate the time-frequency quality of the LC. Figure 14(a) shows that the conventional EMD with a small number of sifting steps is unable to separate the HF components into meaningful IMFs; in addition, it will introduce many LF oscillations that should be excluded. 

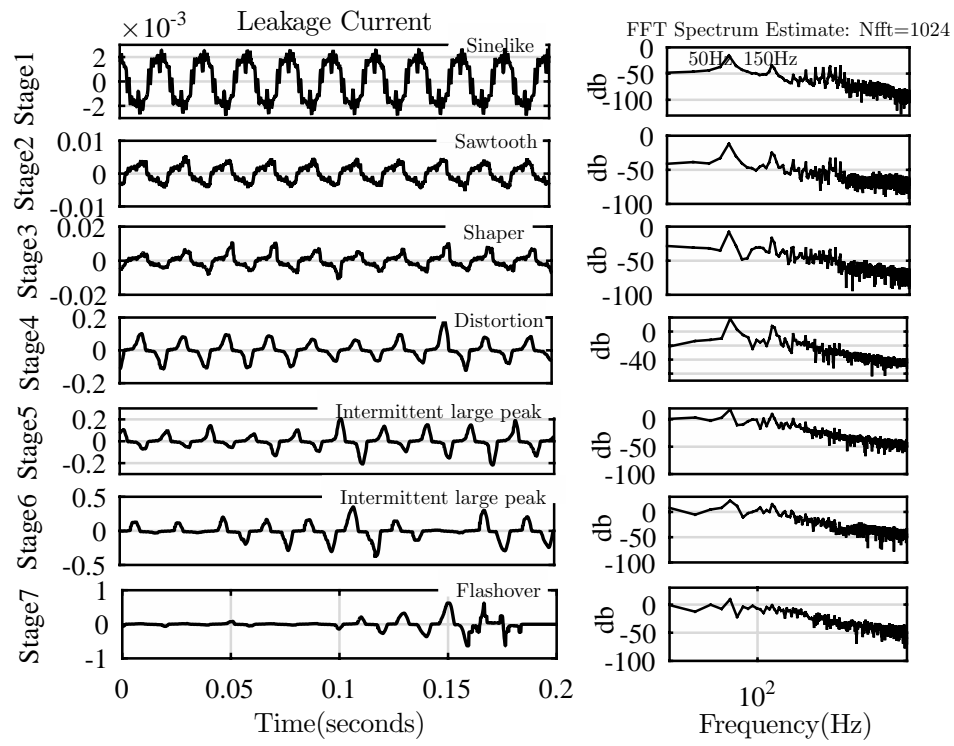

Fig. 12. Transition of LC waveforms and their frequency characteristics. The FT cannot enable us to investigate problems simultaneously in both time and frequency domains, and the FT requires many additional harmonics to simulate nonstationary data because it uses a stationary trigonometric function as an a priori basis [Huang et al. (1998)].
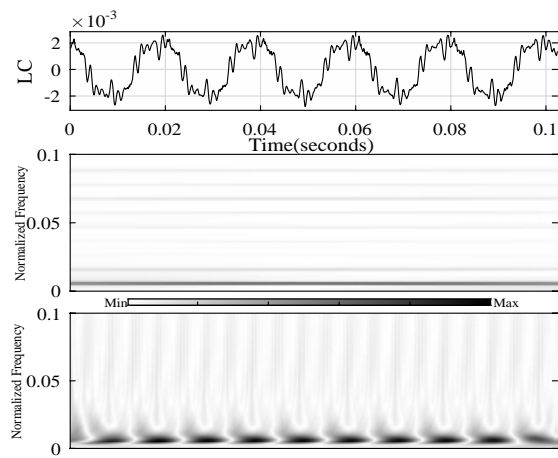

(a) (a)

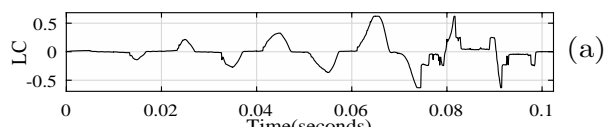

(b)

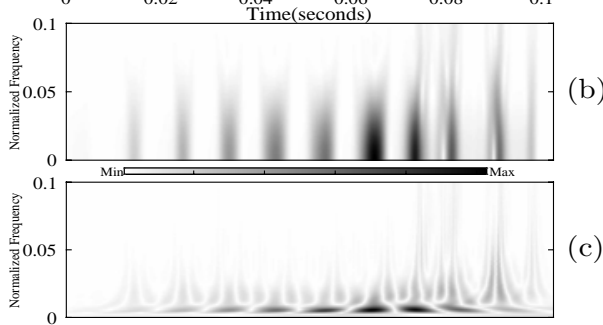

(b)

Fig. 13. Time-frequency analysis of LCs at stage1 and before flashover, (a) original signals, (b) and (c) represent TF property upon the STFT and WT with different window sizes and mother wavelets. (a) TF analysis of stationary LC and (b) TF analysis of nonstationary LC

EMD with fixed sifting iterations and a new stopping criterion is presented in Fig. 14(b), and the separation result is reliable. Considering the EMD-based SPWVD (Fig. 15), stationary LC is a type of weak signal with a fundamental component $(50 \mathrm{~Hz})$ distorted by nonstationary odd harmonics (most likely 3rd harmonic) and noise. 


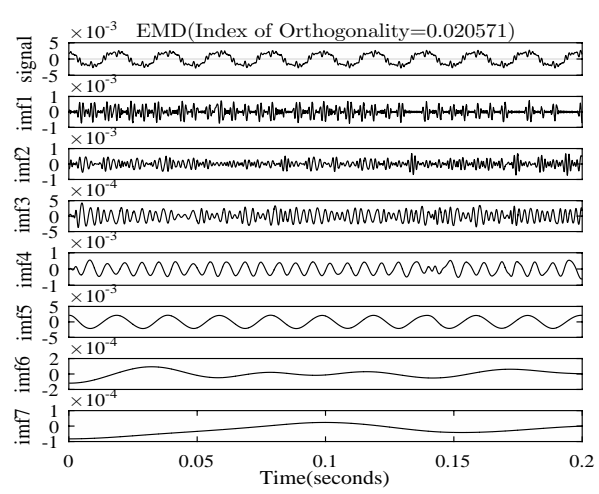

(a)

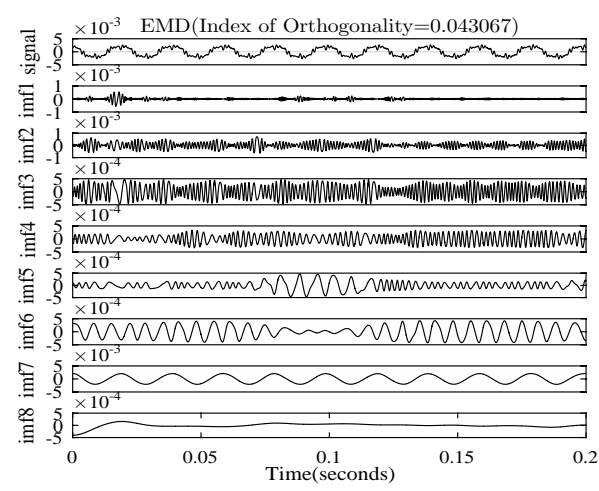

(b)

Fig. 14. EMD of the LC at stage 1 with different sifting iterations. (a) EMD with the mean value criterion and (b) EMD with 500 times of sifting.

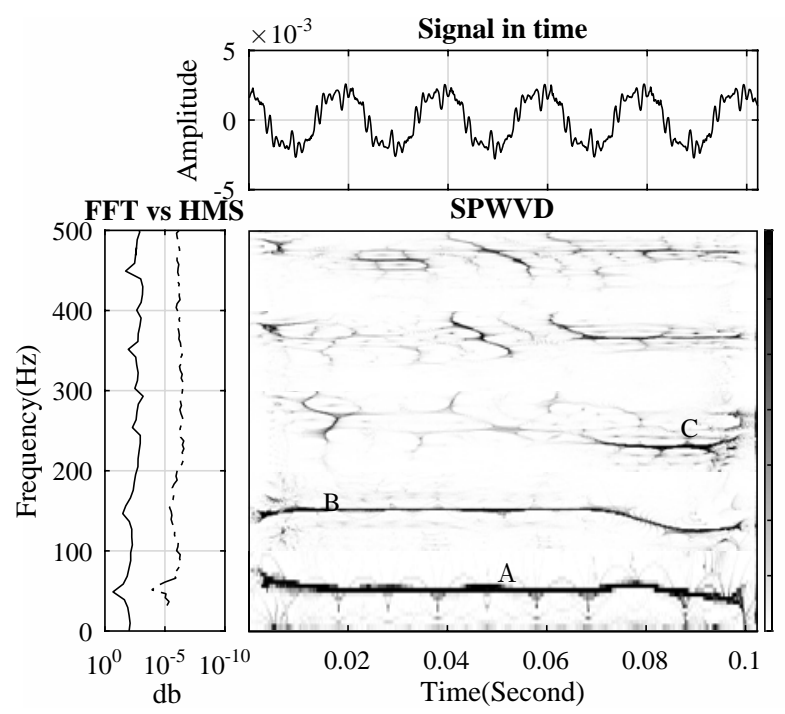

Fig. 15. EMD-based SPWVD of LC at stage 1 (last 6 vibration modes). The letter A denotes the fundamental harmonic, whereas $\mathrm{B}$ and $\mathrm{C}$ represent the 3rd and 5 th harmonics, respectively. Left: FFT spectrum and Hilbert marginal spectrum estimate of the signal (solid line: FFT, dashed line: HMS).

Conventional EMD with a small number of sifting steps is employed to decompose the LC before flashover (Fig. 16(a)); because of the large number of impulses and intermittencies, the EMD will lead to mode mixing and the problem will increase to influence the last IMFs due to the sifting process. In contrast, Fig. 16(b) demonstrates the use of a new masking signal to alleviate this problem, and the result is more reliable. Referring to the EMD-based SPWVD (Fig. 17), LC before flashover is a type of sharply distorted signal with a discontinuous fundamental 


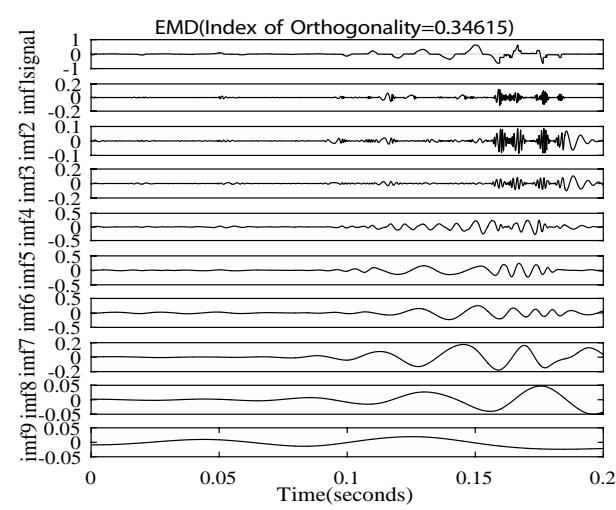

(a)

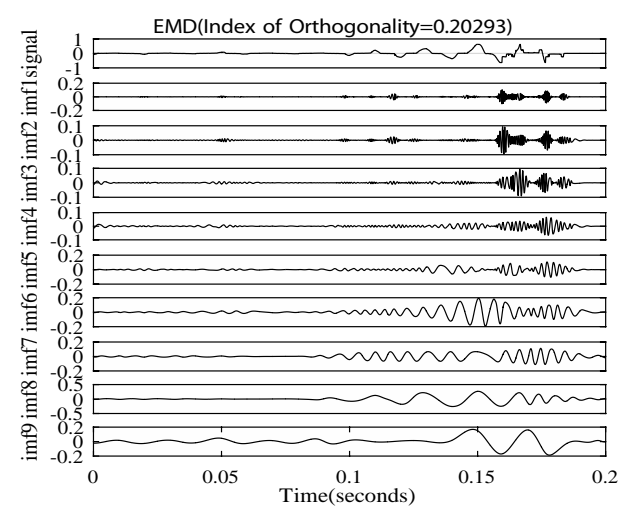

(b)

Fig. 16. EMD of LC before flashover with different sifting iterations. (a) EMD with the mean value criterion and (b) EMD with 500 sifting iterations.

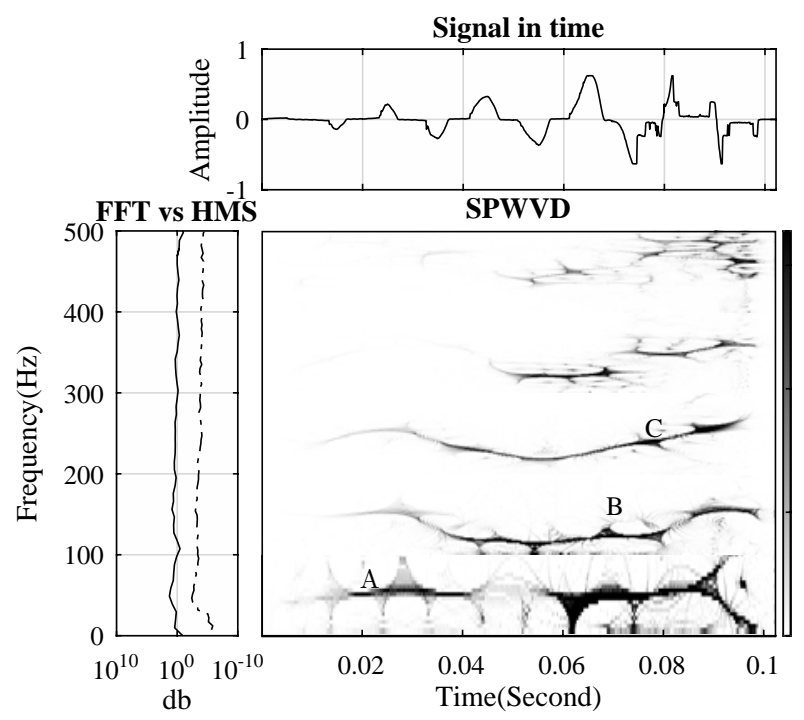

Fig. 17. EMD-based SPWVD of LC before flashover (last 6 vibration modes). The letter A denotes the fundamental harmonic, whereas B and $\mathrm{C}$ represent the 3rd and 5th harmonics, respectively. Left: FFT spectrum and Hilbert marginal spectrum estimate of the signal (solid line: FFT, dashed line: HMS).

component and some odd harmonics with time-varying frequencies; moreover, the $\mathrm{HF}$ components are useful for precisely detecting the flashover point and duration.

\section{Discussions and Conclusions}

(1) Theoretically, the EMD can achieve an arbitrarily high frequency resolution when working with a two-tone signal with the same amplitude; in other words, 
as long as the multi-component signal does not have a rigorous zero-local-mean, a large number of sifting iterations could pick up all components at the cost of information leakage because a function cannot have a maximum and minimum at the same point [Wang et al. (2010)]. (2) The relationship between sifting iterations and resolution ratio follows an exponential distribution; if the frequency ratio under a particular sifting iteration is known, one can obtain all frequency ratios within the specific interval, but there is a trade-off between frequency resolution and information leakage. An optimal number of sifting iterations is required to obtain the best IMFs. (3) Unlike the prism property of the FT and the mathematical microscope property of the WT, the ultimate goal of the HHT is to work as raindrops, through which some light can be represented as a mixture of all the colors of the rainbow. (4) Whether an IMF is a weak or strong mono-component is determined by its extremum distribution. Mathematically, for a multi-component signal, only when EMD can detect all extrema about a specific component can it distinguish the component; moreover, for a mono-component signal, if EMD can find different types of extrema, it will decompose this signal into stronger mono-components. (5) A monocomponent signal is not necessarily a perfect IMF, but a perfect IMF is more likely to be a mono-component signal. However, there is still no rigorously mathematical standard to determine whether a typically defined IMF is a mono-component or multi-component signal or the "watershed" between mono-component and multicomponent IMFs, similar to the circumstance of conductors and insulators. (6) When a two-tone signal has a large frequency ratio and the LF component has higher amplitude at the same time, through the sifting process, this signal will behave as a combination of FM signals, harmonics and a constant; one can only obtain the correct result by using the frequency heterodyne or single-phase DQ techniques. (7) Using the new masking signal is helpful to alleviate the mode mixing problem and distinguish the intermittencies, but a mode mixing phenomenon may still exist in the LF IMFs if the distorted signals have widespread frequencies; thus, additional masking signals with frequencies outside one octave are needed.

Simulation and real-world data are provided to demonstrate the validity of the novel method. The following conclusions can be drawn by employing EMD-based SPWVD and conventional TF methods: 1. The improved EMD-based SPWVD yields not only a more precise frequency resolution and higher temporal resolution in identifying the intra-wave FM signal with few auto-terms than other time-frequency techniques, but also has more physical meaning when studying on signals with time-varying frequencies and with frequencies within one octave (such as LC). 2. The stationary LC waveform behaves as a type of weak signal with a fundamental component distorted by nonstationary odd harmonics (most likely 3rd harmonic) and noise 3. LC before flashover is more likely to have nonlinear features due to large-amplitude arcing, and the waveform is a type of sharply distorted signal with a discontinuous fundamental component and some odd harmonics with time-varying frequencies; moreover, the HF components are useful for precisely detecting the flashover point and flashover duration. 


\section{References}

Afroni, M., Sutanto, D. and Stirling, D. (2013). Analysis of nonstationary power-quality waveforms using iterative Hilbert-Huang transform and SAX algorithm. IEEE Trans. Power Deliv. 28: 2134-2144.

Auger, F., Flandrin, P., Lemoine, O. and Gonçalves, P. (1998). Time-frequency toolbox for Matlab. Available at: http://tftb.nongnu.org.

Bedrosian, E. (1963). A product theorem for Hilbert transforms. Proc. IEEE, 51: 868-869.

Boashash, B. (1992a). Estimating and interpreting the instantaneous frequency of a signal I: Fundamentals. Proc. IEEE, 80: 520-538.

Boashash, B. (1992b). Estimating and interpreting the instantaneous frequency of a signal II: Algorithms and applications. Proc. IEEE, 80: 540-568.

Boashash, B. (1992c). Time-frecuency Signal Analysis: Methods and Applications. Longman Cheshire, Melbourne and John Wiley Halsted Press, New York.

Boudraa, A.-O. and Cexus, J.-C. (2007). EMD-based signal filtering. IEEE Trans. Instrum. Meas., 56: 2196-2202.

Buckheit, J., Chen, S., Donoho, D., Johnstone, I. and Scargle, J. (2005). Wave-lab850. Available at: http://statweb.stanford.edu/ wavelab/.

Daubechies, I., Lu, J. and Wu, H.-T. (2009). Synchrosqueezed wavelet transforms: A tool for empirical mode decomposition. arXiv:0912.2437 [math.NA].

Deering, R. and Kaiser, J. (2005). The use of a masking signal to improve empirical mode decomposition. IEEE Int. Conf. Acoustics, Speech, and Signal Processing (ICASSP '05), Vol. 4, pp. 485-488.

Deléchelle, E., Lemoine, J. and Niang, O. (2005). Empirical mode decomposition: An analytical approach for sifting process. IEEE Signal Process. Lett., 12: 764-767.

Diop, E. H. S., Alexandre, R. and Boudraa, A.-O. (2010). Analysis of intrinsic mode functions: A PDE approach. IEEE Signal Process. Lett., 17: 398-401.

Feldman, M. (2011). Hilbert Transform Applications in Mechanical Vibration. John Wiley \& Sons, p. 750 .

Flandrin, P., Auger, F., Chassande-Mottin, E. et al. (2002). Time-frequency reassignment from principles to algorithms. Applications in Time-Frequency Signal Processing, Vol. 5. CRC Press, pp. 179-203.

Flandrin, P., Rilling, G. and Goncalves, P. (2004). Empirical mode decomposition as a filter bank. IEEE Signal Process. Lett., 11: 112-114.

Gao, H., Mao, Y., Jia, Z. and Guan, Z. (2006). Time and frequency analysis on leakage current waveforms of discharges along hydrophobic and hydrophilic surfaces. Proc. 8th Int. Conf. Properties and Applications of Dielectric Materials (ICPADM), Vol. 2, pp. 595-598.

Hahn, S. L. (1996). Hilbert Transforms in Signal Processing. Artech House, Boston.

Huang, N. E. (2014). Hilbert-Huang Transform and Its Applications, World Scientific, Singapore.

Huang, N. E. and Attoh-Okine, N. O. (2005). The Hilbert-Huang Transform in Engineering. CRC Press.

Huang, N. E., Chen, X., Lo, M.-T. and Wu, Z. (2011). On Hilbert spectral representation: A true time-frequency representation for nonlinear and nonstationary data. $A d v$. Adapt. Data Anal., 3: 63-93.

Huang, N. E. and Shen, S. S. (2005). Hilbert-Huang Transform and Its Applications. World Scientific, Singapore.

Huang, N. E., Shen, Z. and Long, S. R. (1999). A new view of nonlinear water waves: The Hilbert spectrum. Annu. Rev. Fluid Mech., 31: 417-457. 
Huang, N. E., Shen, Z., Long, S. R., Wu, M. C., Shih, H. H., Zheng, Q., Yen, N.-C., Tung, C. C. and Liu, H. H. (1998). The empirical mode decomposition and the Hilbert spectrum for nonlinear and non-stationary time series analysis. Proc. R. Soc. Lond. A, 454: 903-993.

Huang, N. E., Wu, M.-L. C., Long, S. R., Shen, S. S., Qu, W., Gloersen, P. and Fan, K. L. (2003). A confidence limit for the empirical mode decomposition and Hilbert spectral analysis. Proc. R. Soc. Lond. A, 459: 2317-2345.

Huang, N. E. and Wu, Z. (2008). A review on Hilbert-Huang transform: Method and its applications to geophysical studies. Rev. Geophys., 46: RG2006.

Huang, N. E., Wu, Z., Long, S. R., Arnold, K. C., Chen, X. and Blank, K. (2009). On instantaneous frequency. Adv. Adapt. Data Anal., 1: 177-229.

Kvedalen, E. (2003). Signal processing using the Teager Energy Operator and other nonlinear operators, Ph.D. thesis, University of Oslo.

Laila, D. S., Messina, A. R. and Pal, B. C. (2009). A refined Hilbert-Huang transform with applications to interarea oscillation monitoring. IEEE Trans. Power Syst., 24: 610-620.

Linderhed, A. (2009). Image empirical mode decomposition: A new tool for image processing. Adv. Adapt. Data Anal., 1: 265-294.

Majumdar, N. S. (2007). Signal analysis with Huang-Hilbert transforms, Dissertations \& Theses - Gradworks, George Mason University.

Mandic, D. P., Wu, Z., Huang, N. E. et al. (2013). Empirical mode decomposition-based time-frequency analysis of multivariate signals: The power of adaptive data analysis. IEEE Signal Process. Mag., 30: 74-86.

Moula, B., Mekhaldi, A., Teguar, M. and Haddad, A. (2013). Characterization of discharges on non-uniformly polluted glass surfaces using a wavelet transform approach. IEEE Trans. Dielectr. Electr. Insul., 20: 1457-1466.

Norddin, N., Abdullah, A., Abidin, N., Aman, A. and Ramani, A. (2013). Polymeric insulation surface condition analysis using linear time-frequency distributions. IEEE Conf. Power Engineering and Optimization, pp. 558-563.

Nuttall, A. (1966). On the quadrature approximation to the Hilbert transform of modulated signals. Proc. IEEE, 54: 1458-1459.

Qian, T. (2010). Intrinsic mono-component decomposition of functions: An advance of Fourier theory. Math. Methods Appl. Sci., 33: 880-891.

Riemenschneider, S., Liu, B., Xu, Y. and Huang, N. E. (2005). B-spline based empirical mode decomposition. Hilbert-Huang Transform and Its Applications, Vol. 2. World Scientific, Singapore, pp. 27-55.

Rilling, G. and Flandrin, P. (2008). One or two frequencies? The empirical mode decomposition answers. IEEE Trans. Signal Process., 56: 85-95.

Rilling, G., Flandrin, P., Goncalves, P. et al. (2003). On empirical mode decomposition and its algorithms. Proc. IEEE-EURASIP Workshop on Nonlinear Signal and Image Processing, NSIP-03, Grado (I), pp. 8-11.

Rilling, G. and Goncalves, P. (2008). EMD toolbox for Matlab. Available at: http://perso.ens-lyon.fr/patrick.flandrin/emd.html.

Salvino, L. W., Pines, D. J., Todd, M. and Nichols, J. M. (2005). EMD and instantaneous phase detection of structural damage. Hilbert-Huang Transform and Its Applications, Vol. 11. World Scientific, Singapore, pp. 227-262.

Senroy, N., Suryanarayanan, S. and Ribeiro, P. (2007). An improved Hilbert-Huang method for analysis of time-varying waveforms in power quality. IEEE Trans. Power Syst., 22: 1843-1850. 
Sharpley, R. C. and Vatchev, V. (2006). Analysis of the intrinsic mode functions. Constr. Approx. 24: 17-47.

Suda, T. (2005). Frequency characteristics of leakage current waveforms of a string of suspension insulators. IEEE Trans. Power Deliv. 20: 481-487.

Tary, J. B., Herrera1, R. H., Han, J. and van der Baan, M. (2014). Spectral estimation What is new? What is next? Rev. Geophys., 52: 723-749.

Wang, G., Chen, X.-Y., Qiao, F.-L., Wu, Z. and Huang, N. E. (2010). On intrinsic mode function. Adv. Adapt. Data Anal., 2: 277-293.

Wu, Z. and Huang, N. E. (2004). A study of the characteristics of white noise using the empirical mode decomposition method. Proc. R. Soc. Lond. A, 460: 1597-1611.

Wu, Z. and Huang, N. E. (2005). Statistical significance test of intrinsic mode functions. Hilbert-Huang Transform and Its Applications, Vol. 5. World Scientific, Singapore, pp. $107-127$.

Wu, Z. and Huang, N. E. (2009). Ensemble empirical mode decomposition: A noise-assisted data analysis method. Adv. Adapt. Data Anal., 1: 1-41.

$\mathrm{Wu}, \mathrm{Z}$. and Huang, N. E. (2010). On the filtering properties of the empirical mode decomposition. Adv. Adapt. Data Anal., 2: 397-414.

Wu, Z., Huang, N. E. and Chen, X. (2009). The multi-dimensional ensemble empirical mode decomposition method. Adv. Adapt. Data Anal., 1: 339-372.

Yeh, J.-R., Shieh, J.-S. and Huang, N. E. (2010). Complementary ensemble empirical mode decomposition: A novel noise enhanced data analysis method. Adv. Adapt. Data Anal., 2: 135-156. 